\title{
COMPORTAMENTO DE ÍONS DE CHUMBO ASSOCIADOS A ATERROS DOMÉSTICOS
}

\section{BEHAVIOUR OF LEAD ION IN DOMESTIC LANDFILL}

\author{
Claudia Lucia de Moura $^{1}$ e Raphael Hypólito ${ }^{2}$
}

\begin{abstract}
RESUMO Este trabalho foi desenvolvido em área que comporta três aterros domésticos onde se constatou que os sistemas água subterrânea, sedimento de fundo e solo encontram-se seriamente comprometidos por íons chumbo, especialmente por fontes oriundas dos aterros. Em alguns pontos amostrados, os resultados analíticos do chumbo na água subterrânea, foram quatro vezes superiores aos permitidos pelos órgãos oficiais. O solo, graças as propriedades desfavoráveis à retenção catiônicas, em especial do chumbo, constitui-se em mais um fator de risco para o comprometimento ambiental da área. As análises obtidas por Difração de Raios X mostram predominância de caulinita, argilomineral 1:1, com pouco poder de adsorção. Baixos valores de capacidade de troca catiônica e teores pouco significativos de matéria orgânica, também não apresentam características propícias para adsorver íons metálicos, facilitando, desta forma, a disponibilidade de metais pesados ao meio ambiente. Por outro lado, é importante salientar que a população vizinha, praticamente em toda circunvizinhança dos aterros, utiliza para consumo água de poços tipo cacimba, além do uso dessa água em intensas e contínuas atividades hortifrutigranjeiras e agropecuárias.
\end{abstract}

Palavras-chave: aterro sanitário, resíduo sólido, chumbo

\begin{abstract}
This work was performed in an area with three domestic landfills where the groundwater, sediments and soil are seriously contaminated due to the ions of lead, most likely because of the landfill. In some sampling points, results from chemical analysis of groundwater were four times greater than the criteria established by environmental agencies. The soil, due to its unfavorable capability to hold lead, is other risk factor to environmental disturbance in the area. Analysis by Diffraction of X ray showed the tendency of caulinite and gibbsite, which combined with a low valor of ionized change capacity and organic matter, makes the soil not able to absorbed metal ion. On the other hands, to all these factors is added the human activities in the neighborhood. The population uses water from a shallow drinking well and besides there is also farming and vegetable grown in the surroundings.
\end{abstract}

Keywords: domestic landfill, solid waste, lead

\section{INTRODUÇÃO}

O crescimento acelerado das sociedades consumistas, opostas ao desenvolvimento sustentável, tem contribuído de modo significativo para aumento da produção de resíduos que, por muitos anos, não foram descartados em locais apropriados, formando ciclos de agressão ao meio ambiente. Segundo a Associação Brasileira de Empresas de Limpeza Pública e Resíduos Especiais (ABRELPE, 2004), cerca de 8.000 locais de disposição inadequada de resíduos estão, de uma forma ou outra, contaminando o solo e o lençol freático. No Brasil, em 2004, a geração foi cerca de 162.230 t dia $^{-1}$ de resíduos sólidos urbanos, número que vem crescendo a cada ano (ABRELPE, 2004).

Quando se trata de resíduos, o objetivo maior é sua eliminação ou redução ainda na fonte; caso sua geração não possa ser evitada, são indicadas reciclagem, reutilização ou recuperação (STRAUS; MENESES, 1994; RHYNER et al., 1995). No Brasil, devido o baixo custo, com exceção dos lixões, os aterros sanitários ainda são o destino mais comum de resíduos (TENÓRIO; ESPINOSA, 2004). Os aterros sanitários destinados não perigosos, quando construídos e gerenciados de forma apropriada, são boas soluções imediatas de descartes, entretanto, a longo prazo, podem gerar sérios e graves problemas.

Independente da classificação do resíduo, a preocupação de monitoramento em áreas destinadas à sua disposição final, deve ter igual importância, visto que parte do material perigoso continua sendo disposto de forma incorreta, muitos deles misturados aos não perigosos e destinados a aterros nem sempre adequados para receber materiais dessa natureza. O estudo do comportamento de íons de metais pesados associados aos locais de descarte de resíduos e seu entorno é imprescindível para detecção e controle da poluição ambiental.

\footnotetext{
${ }^{1}$ Programa de Pós-Graduação em Geociências Instituto de Geociências - IGc/USP (claudiamoura@igc.usp.br)

${ }^{2}$ Instituto de Geociências - IGc/USP (hypolito@usp.br)
} 
Neste trabalho é estudado especialmente o comportamento do íon chumbo, um dos metais pesados freqüentemente associados aos locais de descartes de resíduos e que pode, devido sua periculosidade, levar a sérios comprometimentos ao meio ambiente e à saúde das pessoas.

O comportamento do chumbo em água depende essencialmente dos parâmetros químicos e físico-químicos do meio como, por exemplo, condições de $\mathrm{pH}$, potenciais de óxido-redução (Eh), presença de matéria orgânica, agentes complexantes, etc. Desta forma ele pode permanecer em solução, dissolvido ou precipitarse como óxidos, hidróxidos ou carbonatos (ATSDR, 1999).

O acúmulo de chumbo no solo ocorre principalmente em função da taxa de deposição da atmosfera, ficando, em sua maior parte, retido em suas camadas superficiais podendo, eventualmente, ser transportado para águas superficiais e subterrâneas. Os fatores que podem auxiliar no transporte e disponibilidade do chumbo no solo são os valores de $\mathrm{pH}$, composição mineral, matéria orgânica, presença de colóides de óxidos de ferro e capacidade de troca iônica (WHO, 1981).

No solo, de modo geral, as concentrações naturais de chumbo são baixas. Valores de referência para solos e águas subterrâneas foram sugeridos pela CETESB (2005), respectivamente $17 \mathrm{mg} \mathrm{kg}^{-1}$ e $10 \mu \mathrm{g} \mathrm{L}^{-1}$. Existem ainda os valores de intervenção que indicam existência de contaminação do solo ou água subterrânea e se baseiam na avaliação de risco, tendo caráter corretivo. Os valores de intervenção para solos agrícolas, residenciais e industriais foram, respectivamente, estabelecidos em 180, 300 e $900 \mathrm{mg} \cdot \mathrm{kg}^{-1}$ (CETESB, 2005, op. cit). Para sedimentos a CETESB utiliza valores internacionais de 35 e $91,3 \mathrm{mg} \mathrm{kg}^{-1}$ Interim Sediment Quality Guideline (ISQG) e Probable Effect Level (PEL), respectivamente, sugeridos por Smith et al. (1996).

\section{ÁREA DE ESTUDO}

A área de estudo está localizada no Município de Tremembé, Estado de São Paulo, pertencente à Região do Vale do Paraíba. No contexto geológico, a área pertence à Bacia de Taubaté situada no Complexo Cristalino Pré-
Cambriano do Leste Paulista, no Bloco Tectônico Paraíba do Sul, limitados pela Falha do Buquira ao norte, e ao sul pela Falha do Alto da Fartura. Outras falhas de menor porte são reconhecidas nessa região, como as Falhas da Serra do Jambeiro, Bom Retiro, Pinheiros, Ronco, entre outras (HASUI; PONÇANO, 1978).

O tipo de solo predominante na região é classificado como Podzólicos e Latossolo Vermelho e Amarelo (EMBRAPA, 1981). Seus usos predominantes são aqüicultura e avicultura, pecuária, principalmente leiteira e rizicultura, sendo a maioria das propriedades de pequeno e médio portes.

O regime hídrico é caracterizado como Perúdico (com muita chuva) e o regime térmico dos solos classifica-se como isohipertérmico com profundidade média de $50 \mathrm{~cm}$. $O$ índice pluviométrico anual acha-se em torno de 1.430 mm (WAMBEKE, 1981).

O clima é considerado mesotérmico com temperaturas médias anuais inferiores a $18{ }^{\circ} \mathrm{C}$ nos meses frio (junho e julho) e superiores à $25{ }^{\circ} \mathrm{C}$ nos meses quentes (janeiro e fevereiro), resultando na média anual de $24,7^{\circ} \mathrm{C}$.

\section{MATERIAL E MÉTODOS}

Os trabalhos de campo consistiram em coletas de amostras de solos, sedimentos de fundo dos leitos dos rios, águas subterrâneas e superficiais.

Na figura 1 é apresentado mapa esquemático da área de estudos, assim como localização dos pontos de amostragem e direções do fluxo da água subterrânea.

Os pontos amostrados abrangem três aterros que, segundo a atual classificação de resíduos (NBR 10004, 2004), são enquadrados na classe IIB (ATERROS A e B) e classe IIA (ATERRO C). Em cada um deles foram perfurados poços de monitoramento cadastrados como $\mathrm{A}_{\mathrm{A} 1}, \mathrm{~A}_{\mathrm{A} 2}, \mathrm{~B}_{\mathrm{B} 1}$, $\mathrm{B}_{\mathrm{B} 2}, \mathrm{C}_{\mathrm{C} 1}$ e $\mathrm{C}_{\mathrm{C} 2}$ e coletadas águas subterrâneas.

Coletaram-se amostras de águas superficiais do Rio Serragem $\left(R_{a 1}, R_{a 2}\right.$ e $\left.R_{a 3}\right)$ e de um de seus afluentes $\left(R_{a 4}\right)$ que sofre influência direta dos aterros. Também foram coletadas amostras do Rio Pimenta $\left(R_{A 1}\right)$ que se localiza cerca de $4 \mathrm{~km}$ dos aterros, consideradas como referência. 


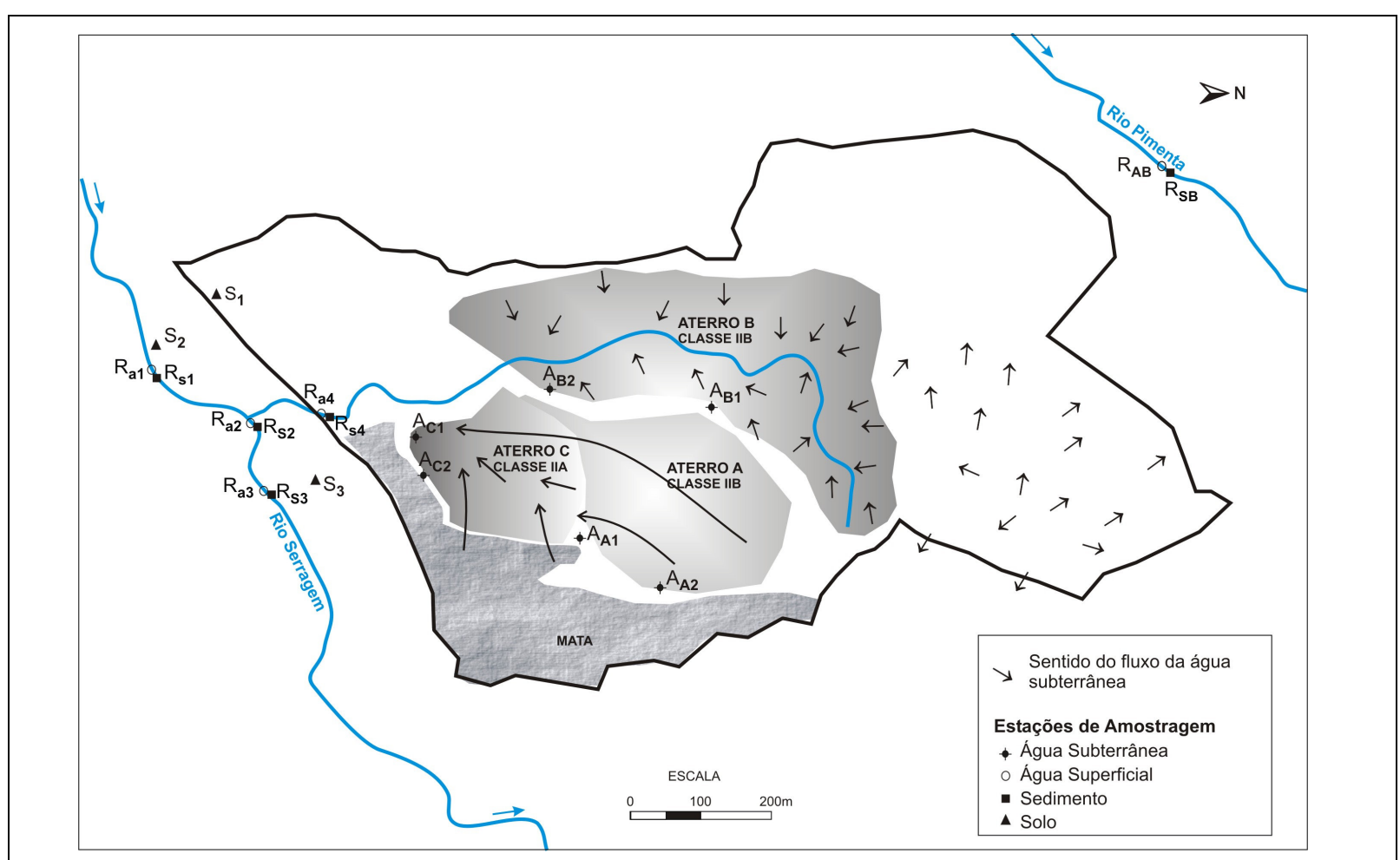

Figura 1 - Área de estudo com os pontos de amostragens e indicação das direções de fluxo da água subterrânea. Figure 1 - Area of study showing the sampling points, and the direction of groundwater flow.

A amostra $S_{1}$ corresponde a antigo leito de secagem de resíduos e as amostras $\mathrm{S}_{2}$ e $\mathrm{S}_{3}$ referem-se, respectivamente, a solos coletados em locais antes e depois da influência dos Aterros. Nos Rios Pimenta $\left(\mathrm{R}_{\mathrm{S} 1}\right)$ e Serragem $\left(\mathrm{R}_{\mathrm{S} 2}\right.$, $R_{S 3}$ e $R_{S 4}$ ) foram ainda coletadas amostras de sedimentos de fundo.

Durante as amostragens foram medidos in situ parâmetros químicos e físico-químicos como temperatura, $\mathrm{pH}$, potencial de óxido-redução (Eh), Condutividade Elétrica (CE), Oxigênio Dissolvido (OD) e Alcalinidade (HYPOLITO et al., 2008).

As amostras de solo foram coletadas em diferentes profundidades, perfurando poços com trado manual tipo holandês (diâmetro de 3"') e, as de sedimentos de fundo foram coletadas utilizando tubos de PVC (diâmetro de 3") cravados nos leitos dos rios.

As amostras das águas foram filtradas (filtro de $0,45 \mu \mathrm{m})$, acidificadas $\left(\mathrm{HNO}_{3}\right)$ e destinadas às análises químicas por Espectrofotômetro de Absorção Atômica de Chama, modelo 932 B Plus. As amostras de referência, bem como aquelas destinadas às análises químicas de ânions, foram mantidas sem preservantes, a temperaturas próximas de $4{ }^{\circ} \mathrm{C}$, em recipientes termoisolantes e analisadas por Cromatografia Líquida (Dionex 2010 i). Todas as análises foram realizadas nos laboratórios do Centro de Pesquisa de Águas Subterrâneas (CEPAS) do Instituto de
Geociências da Universidade de São Paulo (IGc/USP).

As amostras de solo e sedimentos de fundo foram secas à temperatura ambiente, destorroadas, quarteadas e peneiradas, separando as frações finas inferiores a $2 \mathrm{~mm}$.

As análises granulométricas qualitativas e quantitativas foram realizadas pelos Métodos da Pipeta de Robson (dispersão total) e os resultados foram projetados em diagrama triangular e classificados segundo Shepard (1954).

As amostras de materiais sólidos foram analisadas química e mineralogicamente por Espectrofotometria por Fluorescência de Raios X (FRX) e Difração de Raios X (DRX), respectivamente.

Foram efetuadas também determinações de Capacidade de Troca Catiônica (CTC), pH e matéria orgânica (MO) do solo.

Das diferentes frações do perfil do solo foram extraídos os íons metálicos com $\mathrm{HNO}_{3}$ (extração total, $8 \mathrm{M}$ ) e as soluções ácidas analisadas quimicamente.

\section{RESULTADOS E DISCUSSÃO}

As amostras de águas subterrâneas apresentaram caráter ácido com pH médio 5,90; os potenciais de óxido-redução (Eh) foram sempre positivos, indicando ambientes oxidantes em todos os pontos de amostragem. Os valores de 
pH das águas justificam a forma iônica segundo a qual o chumbo é encontrado.

Os resultados analíticos das águas subterrâneas, nas estações de amostragem $\mathrm{A}_{\mathrm{Al}}$, $\mathrm{A}_{\mathrm{A} 2}$ e $\mathrm{A}_{\mathrm{C} 2}$, indicaram para $\mathrm{o}$ chumbo concentrações quatro vezes acima dos limites permitidos pela CETESB (Tabela 1). Este fato se reveste de grande preocupação de caráter ambiental, uma vez que na região a grande maioria da população se serve de águas extraídas de poços do tipo cacimba e possuem intensa atividade de natureza hortifrutigranjeira e agropecuária, além de criação de rãs.

As águas superficiais apresentaram caráter ligeiramente básico, próximo à neutralidade, com pH médio 7,70 e potenciais de óxido-redução (Eh) positivos.
A condutividade elétrica foi excepcionalmente elevada nos pontos $R_{\mathrm{a} 2}$ e principalmente em $R_{a 4}$ mais próximos aos aterros, apresentando concentrações elevadas de sódio, sulfatos e cloretos (Tabela 2).

Os resultados das análises químicas, mineralógicas, assim como os dados de Difração de Raios X, Capacidade de Troca Catiônica e pH do solo encontram-se nas tabelas 3 e 4 .

Os valores positivos de $\Delta \mathrm{pH}$ do solo indicam presença dominante de cargas negativas nas superfícies das partículas do solo, aptas a adsorverem íons metálicos. A presença predominante de caulinita (argilomineral 1:1, de baixo poder de adsorção) justifica os baixos valores de CTC.

Tabela 1-Parâmetros químicos, físico-químicos e resultados analíticos das águas subterrâneas dos aterros A, B e C. Table 1 - Chemical and physical-chemical parameters and groundwater data from Landfill A, B and C.

\begin{tabular}{|c|c|c|c|c|c|c|}
\hline Parâmetros & $\mathrm{A}_{\mathrm{A} 1}$ & $\mathrm{~A}_{\mathrm{A} 2}$ & $\mathrm{~A}_{\mathrm{B} 1}$ & $\mathrm{~A}_{\mathrm{B} 2}$ & $\mathrm{~A}_{\mathrm{C} 1}$ & $\mathrm{~A}_{\mathrm{C} 2}$ \\
\hline $\mathrm{pH}$ & 5,64 & 5,95 & 5,92 & 6,08 & 6,73 & 6,44 \\
\hline Eh $(\mathrm{V})$ & $+0,16$ & $+0,25$ & $+0,23$ & $+0,21$ & $+0,12$ & $+0,29$ \\
\hline $\mathrm{CE}\left(\mathrm{mS} \mathrm{cm}^{-1}\right)$ & 62 & 89 & 50 & 19 & 45 & 18 \\
\hline Alcalinidade $\left(\mathrm{CaCO}_{3} \mathrm{mg} / \mathrm{dm}^{-3}\right)$ & 13,00 & 8,00 & 11,00 & 3,00 & 8,00 & 8,00 \\
\hline $\mathrm{Pb}^{2+}\left(\mathrm{mg} / \mathrm{dm}^{-3}\right)$ & 0,04 & 0,04 & nd & nd & nd & 0,04 \\
\hline $\mathrm{Na}^{+}\left(\mathrm{mg} / \mathrm{dm}^{-3}\right)$ & 4,70 & 6,70 & 4,00 & 1,70 & 3,50 & 0,80 \\
\hline $\mathrm{K}^{+}\left(\mathrm{mg} / \mathrm{dm}^{-3}\right)$ & 5,50 & 2,70 & 4,70 & 5,90 & 2,10 & 4,30 \\
\hline $\mathrm{Ca}^{2+}\left(\mathrm{mg} / \mathrm{dm}^{-3}\right)$ & 2,90 & 11,75 & 3,70 & 0,65 & 1,95 & 9,95 \\
\hline $\mathrm{Mg}^{2+}\left(\mathrm{mg} / \mathrm{dm}^{-3}\right)$ & 0,40 & 4,10 & 0,80 & 0,60 & 0,50 & 2,50 \\
\hline $\mathrm{Cl}^{-}\left(\mathrm{mg} / \mathrm{dm}^{-3}\right)$ & 0,27 & 0,97 & 0,12 & 0,57 & 0,22 & 0,38 \\
\hline $\mathrm{NO}_{3}{ }^{-}\left(\mathrm{mg} / \mathrm{dm}^{-3}\right)$ & 1,57 & 7,23 & 1,34 & 2,0 & 5,48 & 0,96 \\
\hline $\mathrm{SO}_{4}{ }^{2-}\left(\mathrm{mg} / \mathrm{dm}^{-3}\right)$ & 68,89 & 1,34 & 0,25 & 0,25 & 0,17 & 2,31 \\
\hline
\end{tabular}

nd = não detectado

Tabela 2 - Parâmetros físico-químicos e dados analíticos das águas superficiais dos aterros A, B e C.

Table 2 - Chemical and physical-chemical parameters and surface waters analytical data from Landfill A, $B$ and $C$.

\begin{tabular}{l|c|c|c|c|c}
\hline Parâmetros & & & $\mathrm{R}_{\mathrm{a} 2}$ & $\mathrm{R}_{\mathrm{a} 3}$ & $\mathrm{R}_{\mathrm{a} 4}$ \\
\hline $\mathrm{pH}$ & $\mathrm{R}_{\mathrm{AB}}$ & $\mathrm{R}_{\mathrm{a} 1}$ & & & 7,30 \\
\hline $\mathrm{Eh}(\mathrm{V})$ & 8,52 & 8,25 & 8,42 & 5,80 & $+0,12$ \\
\hline $\left.\mathrm{CE}(\mathrm{mS} \mathrm{cm})^{-1}\right)$ & $+0,17$ & $+0,12$ & $+0,19$ & $+0,20$ & 1185 \\
\hline $\left.\mathrm{Alcalinidade}^{-3} \mathrm{CaCO}_{3} \mathrm{mg} / \mathrm{dm}^{-3}\right)$ & 21 & 66 & 250 & 61 & 43,0 \\
\hline $\mathrm{Pb}^{2+}\left(\mathrm{mg} / \mathrm{dm}^{-3}\right)$ & 5,0 & 8,0 & 40,0 & 11,0 & $\mathrm{nd}$ \\
\hline $\mathrm{Na}^{+}\left(\mathrm{mg} / \mathrm{dm}^{-3}\right)$ & $\mathrm{nd}$ & $\mathrm{nd}$ & $\mathrm{nd}$ & $\mathrm{nd}$ & 32,60 \\
\hline $\mathrm{K}^{+}\left(\mathrm{mg} / \mathrm{dm}^{-3}\right)$ & 2,20 & 2,40 & 28,60 & 2,30 & 3,60 \\
\hline $\mathrm{Ca}^{2+}\left(\mathrm{mg} / \mathrm{dm}^{-3}\right)$ & 2,00 & 1,80 & 3,80 & 1,30 & 8,60 \\
\hline $\mathrm{Mg}^{2+}\left(\mathrm{mg} / \mathrm{dm}^{-3}\right)$ & 1,30 & 1,35 & 3,20 & 1,45 & 1,95 \\
\hline $\mathrm{Cl}^{-}\left(\mathrm{mg} / \mathrm{dm}^{-3}\right)$ & 0,45 & 0,55 & 0,45 & 1,50 & 23,36 \\
\hline $\mathrm{NO}_{3}{ }^{-}\left(\mathrm{mg} / \mathrm{dm}^{-3}\right)$ & 0,65 & 1,38 & 24,91 & 0,96 & 1,68 \\
\hline $\mathrm{SO}_{4}{ }^{2-}\left(\mathrm{mg} / \mathrm{dm}^{-3}\right)$ & 0,16 & 2,36 & 1,77 & 0,87 & 126,06 \\
\hline
\end{tabular}

nd = não detectado 
Tabela 3 - Resultados das determinações de CTC, pH (cloretos de cálcio e de potássio), $\Delta$ pH e matéria orgânica do solo.

Table 3 - Results from Cationic Exchange Capacity, $p H$ and soil organic matter.

\begin{tabular}{|c|c|c|c|c|c|c|}
\hline $\begin{array}{rr}\text { Pontos amostrados } \\
\text { (Prof.) }\end{array}$ & $\begin{array}{c}S_{1} \\
(0-20 \mathrm{~cm})\end{array}$ & $\begin{array}{c}\mathrm{S}_{1} \\
(20-50 \mathrm{~cm})\end{array}$ & $\begin{array}{c}S_{2} \\
(0-20 \mathrm{~cm})\end{array}$ & $\begin{array}{c}S_{2} \\
(20-45 \mathrm{~cm})\end{array}$ & $\begin{array}{c}\mathrm{S}_{3} \\
(0-20 \mathrm{~cm})\end{array}$ & $\begin{array}{c}\mathrm{S}_{3} \\
(20-40 \mathrm{~cm})\end{array}$ \\
\hline $\mathrm{pH}\left(\mathrm{CaCl}_{2}\right)$ & 4,10 & 4,10 & 6,40 & 4,90 & 6,00 & 5,30 \\
\hline $\mathrm{pH}(\mathrm{KCl})$ & 4,00 & 3,90 & 6,10 & 4,70 & 5,70 & 5,00 \\
\hline$\Delta \mathrm{pH}$ & $+0,10$ & $+0,20$ & $+0,30$ & $+0,20$ & $+0,30$ & $+0,30$ \\
\hline CTC $\left(\mathrm{cmol}_{\mathrm{c}} \mathrm{kg}^{-1}\right)$ & 3,50 & 4,10 & 55,30 & 31,00 & 34,90 & 30,50 \\
\hline Matéria orgânica $\left(\mathrm{mg} / \mathrm{dm}^{-3}\right)$ & 2 & 1 & 8 & 6 & 18 & 6 \\
\hline
\end{tabular}

Tabela 4 - Resultados das análises mineralógicas do solo.

Table 4 - Results from soil mineralogical analysis.

\begin{tabular}{c|l}
\hline Pontos Amostrados & \multicolumn{1}{c}{ Composição mineralógica } \\
\hline $\mathrm{S}_{1}$ & Caulinita $\left.\left(\mathrm{Al}_{2} \mathrm{Si}_{2} \mathrm{O}_{5}(\mathrm{OH})_{4}\right)\right) ;$ \\
\hline $\mathrm{S}_{2}$ & Caulinita; illita $\left(\mathrm{Kal}_{2}\left(\mathrm{Si}_{3} \mathrm{Al}\right) \mathrm{O}_{10}(\mathrm{OH})_{2}\right) ;$ gibbsita $\left(\mathrm{Al}(\mathrm{OH})_{3}\right) ;$ \\
\hline $\mathrm{S}_{3}$ & Caulinita, illita e gibbsita; \\
\hline
\end{tabular}

Tabela 5 - Resultados das análises químicas de íons chumbo dos solos e sedimentos de fundo - FRX (a) e Extrações totais (b)

Table 5 - Results from chemical analysis of $\mathrm{Pb}$ in soil and bottom sediment - FRX (a) and Total Extractions (b)

\begin{tabular}{|c|c|c|c|c|c|c|c|c|c|c|}
\hline Parâmetros & $\mathrm{R}_{\mathrm{S} 1}$ & $\mathrm{R}_{\mathrm{S} 2}$ & $\mathrm{R}_{\mathrm{S} 3}$ & $\mathrm{R}_{\mathrm{S} 4}$ & $\begin{array}{c}\mathrm{S}_{1} \\
(0- \\
20 \mathrm{~cm})\end{array}$ & $\begin{array}{c}\mathrm{S}_{1} \\
(20- \\
50 \mathrm{~cm})\end{array}$ & $\begin{array}{c}\mathrm{S}_{2} \\
(0- \\
20 \mathrm{~cm})\end{array}$ & $\begin{array}{c}\mathrm{S}_{2} \\
(20- \\
45 \mathrm{~cm})\end{array}$ & $\begin{array}{c}\mathrm{S}_{3} \\
(0- \\
20 \mathrm{~cm})\end{array}$ & $\begin{array}{c}\mathrm{S}_{3} \\
(20- \\
40 \mathrm{~cm})\end{array}$ \\
\hline $\mathrm{Pb}\left(\mathrm{mg} \mathrm{kg}^{-1}\right)(\mathrm{a})$ & 19 & 227 & 44 & 43 & 42 & 37 & 44 & 48 & 92 & 40 \\
\hline $\mathrm{Pb}\left(\mathrm{mg} \mathrm{kg}^{-1}\right)(\mathrm{b})$ & 18,23 & 142,58 & 42,48 & 31,68 & 32,95 & 30,90 & 27,86 & 33,08 & 69,77 & 35,98 \\
\hline
\end{tabular}

Os resultados obtidos nas extrações totais quando comparados com os resultados das análises por Fluorescência de raios $\mathrm{X}$ dos materiais sólidos (íons estruturais e adsorvidos), indicam baixa disponibilidade iônica para as espécies químicas, com exceção apenas para o chumbo. Neste caso, nota-se que, em praticamente todas as amostras, a disponibilidade desse cátion é consequiência de atividades antrópicas, uma vez que ele se encontra associado à fase sólida do solo por fenômenos como de adsorção e não estruturalmente (Tabela 5).

As projeções dos dados granulométricos em diagrama de Shepard (Figura 2) dos sedimentos de fundo dos rios e do solo e permitiram que se classificassem as maiorias das amostras como arenosa, areia argilosa e síltica.

Análise dos resultados do solo dos três Aterros permite afirmar que seus atributos (granulometria, mineralogia, matéria orgânica e capacidade de troca catiônica, etc.) não são propícios para retenção iônica, podendo levar a comprometimentos aos recursos hídricos da região.

\section{CONCLUSÕES}

Nos três aterros estudados, os resultados analíticos das águas superficiais, subterrâneas, do solo e sedimentos de fundo dos rios, bem como dos parâmetros medidos em campo, mostram intensa disponibilidade de íons de metais pesados, em especial o chumbo.

Os resultados analíticos das águas subterrâneas indicam a necessidade de contínuo e sistemático monitoramento na área dos aterros, bem como, em suas circunvizinhanças, ainda mais levando em conta que uma porção considerável da população da área se serve de água através de poços tipo cacimba para consumo próprio, para atividades hortifrutigranjeiras, agropecuárias etc.

O estudo dos atributos dos solos onde se localizam os aterros, não oferecendo condições para retenção de íons de metais pesados, permite que se aconselhem cessação de suas atividades e instalações em locais apropriados. 


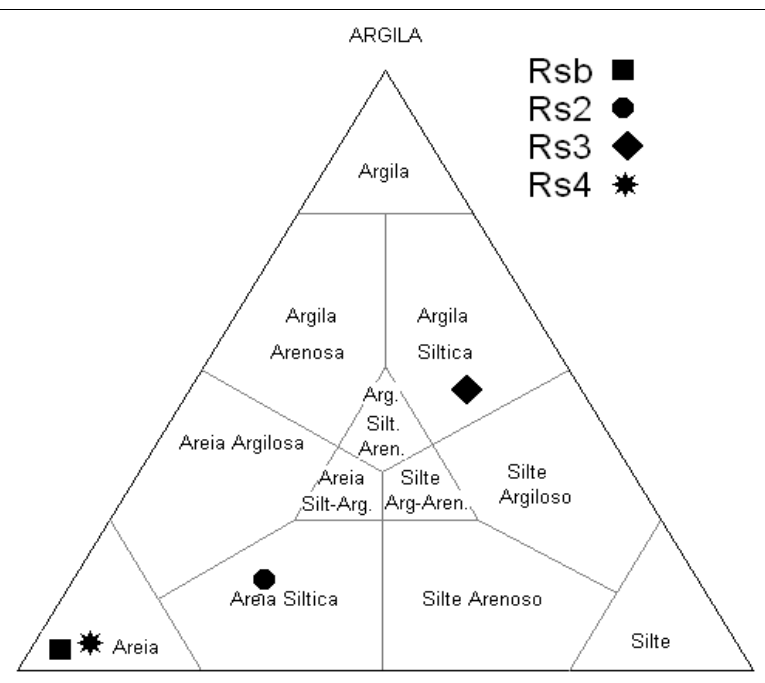

AREIA SILTE

A)

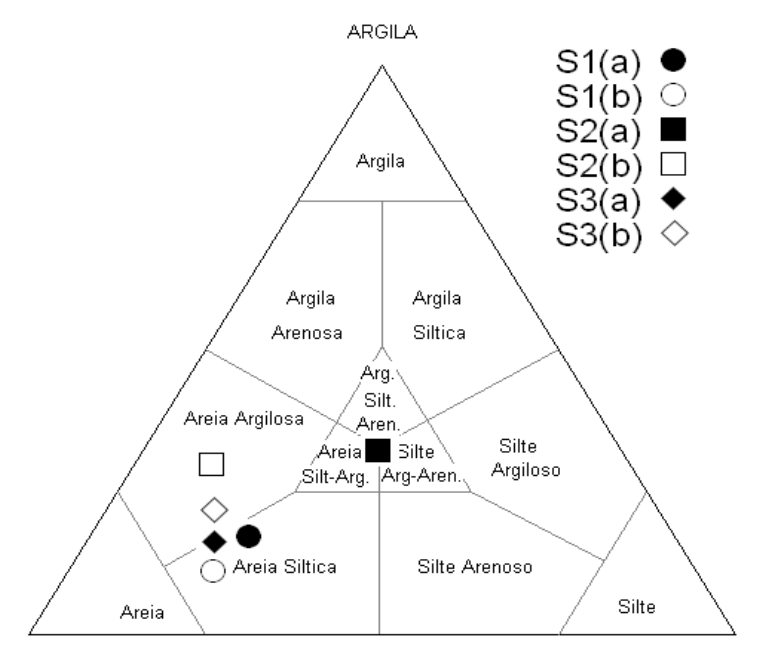

AREIA

SILTE

B)

Figura 2 - Amostras de sedimentos de fundo (a) e solo (b) projetadas em diagramas triangulares de SHEPARD (1954).

Figure 2 - Bottom sediment samples (a) and soil (b) by SHEPARD diagram. 


\section{REFERÊNCIAS}

ABRELPE: Associação Brasileira de Empresas de Limpeza Pública e Resíduos Especiais: Panorama dos resíduos Sólidos no Brasil. Disponível em http://www.abrelpe.com.br. 83 p., 2004.

ABNT-ASSOCIAÇÃO BRASILEIRA DE NORMAS TÉCNICAS- NBR 10004. Resíduos Sólidos: Classificação. 71 p., 2004.

ATSDR-Agency for Toxic Substances and Disease Registry.. Toxicological profile for aluminium and compounds. Atlanta, Georgia. 136 p., 1999.

CETESB - Companhia de Tecnologia de Saneamento Ambiental. Parâmetros para Qualidade de Solo e Água Subterrânea, 2005.

EMBRAPA-EMPRESA BRASILEIRA DE PESQUISA AGROPECUÁRIA. Mapa de solos do Brasil. Serviço Nacional de Levantamento e Conservação de solos. Rio de janeiro, Ministério da Agricultura. 1981.

HASUI, Y.; PONÇANO, W.L. Organização Estrutural e Evolução da Bacia de Taubaté. Cong. Brás. Geol., n. 30. Recife. Anais, SBG. P. 368-381. 1978.

HYPOLITO, R.; ANDRADE， S.; SILVA， L.H.; NASCIMENTO, S.C. Alcalinidade - Metodologia para Determinação em Campo. Analytica. Ano 6, n. 35. p. 52-61. 2008.

MARQUES, J.F. Comportamento de íons $\mathbf{P b}, \mathbf{Z n}$ e Cu em área impactada por escória, produto da reciclagem de baterias chumbo-ácido. 2003. 120 p. Dissertação (Mestrado). Instituto de Geociências, Universidade de São Paulo.

NASCIMENTO S.C. Comportamento de Íons e sua associação a resíduos de indústria siderúrgica em manguezal do sistema estuário de Santos Cubatão/SP. 2007. 112 p. Tese (Doutorado). Instituto de Geociências, Universidade de São Paulo.

PUGAS, M.S. Íons de Metais Pesados $\mathrm{Ni}, \mathrm{Cu}$ e $\mathrm{Cr}$ em Área Impactada por Resíduos de Galvanoplastia na Região Metropolitana de São Paulo-SP. 2007. 85 p. Dissertação (Mestrado). Instituo de Geociências, Universidade de São Paulo.
SHEPARD, F.P. Revised Nomenclature for Depositional Coastal Features. American Association of Petroleum Geololgist Bulletin, Tulsa. V.36, n.10, p.1902-1912, 1954.

RHYNER, C.; SCHWARTZ, L.J.; WENGER, R.B.; KOHRELL, M.G. Waste Management and Resource Recovery. Lewis Publishers. 524 p. 1995.

SMITH, S.L., D.D. MacDonald, K.A. Keenleyside, C.G. Ingersoll; L.J. Field. A Preliminary Evaluation of sediment Quality Assessment Values for Freshwater Ecosystems. Journal of great Lakes Research v.22, n.3, p.624-638, 1996.

STRAUS, E.L.; MENEZES, L.V.T. Minimização de Resíduos. In: CONGRESSO BRASILEIRO DE ENGENHARIA SANITÁRIA E AMBIENTAL, 17, 1994.

TENÓRIO, J.A.S.; ESPINOSA, D.C.R. Controle Ambiental de Resíduos In: Curso de Gestão Ambiental PHILIPPI, A.Jr.; ROMÉRO, M.A.; BRUNA, G.C.; Universidade de São Paulo Faculdade de Saúde Pública, Faculdade de Arquitetura e Urbanismo, Núcleo de Informações em Saúde Ambiental. 2004.

WAMBEKE, V.A. Calculated soil moisture and temperature regimes of South. 1981.

WHO-World Health Organization. Environmental Health Criteria, n.17 Manganese. Geneva. 110p., 1981.

\section{AGRADECIMENTOS}

Os autores agradecem ao Centro de Pesquisas de Águas Subterrâneas (CEPAS), Departamento de Mineralogia e Geotectônica - Laboratórios de Fluorescência e Difração de Raios X, do Instituto de Geociências da Universidade de São Paulo. 
